\title{
Dinâmica espaço-temporal das anomalias de precipitação em uma região semiárida, Nordeste do Brasil
}

\author{
Spatio-temporal dynamics of precipitation anomalies in a \\ semiarid region, Northeastern Brazil
}

\begin{abstract}
Sidney Anderson Teixeira da Costa $^{1}$ (D), Alan Cezar Bezerra ${ }^{1}$ (D), Athos Murilo Queiroz de Araújo $^{2}$ (D), Michelle Ferreira da Silva ${ }^{1}$ (D), José Francisco da Cruz ${ }^{1}$ (D), Rafael Mateus Alves ${ }^{3}$ (i), Luciana Sandra Bastos de Souza ${ }^{1}$ (D)

${ }^{1}$ Universidade Federal Rural de Pernambuco - UFRPE, Serra Talhada, PE, Brasil. E-mails: sidneyeng.agro@outlook.com, cezaralan.a@gmail.com, michellefs2008@gmail.com, zenetto.agronomia@gmail.com, sanddrabastos@yahoo.com.br

${ }^{2}$ Universidade Federal de Pernambuco - UFPE, Caruaru, PE, Brasil. E-mail: athosm.eng@gmail.com

${ }^{3}$ Escola Superior de Agricultura "Luiz de Queiroz" - ESALQ, Universidade de São Paulo - USP, Piracicaba, SP, Brasil. E mail: rafaelalvesmateus@gmail.com
\end{abstract}

\begin{abstract}
Como citar: Costa, S. A. T., Bezerra, A. C., Araújo, A. M. Q., Silva, M. F., Alves, R. M., \& Souza, L. S. B. (2021). Dinâmica espaço-temporal das anomalias de precipitação em uma região semiárida, Nordeste do Brasil. Revista de Gestão de Água da América Latina, 18, e14. https://doi.org/10.21168/rega.v18e14
\end{abstract}

RESUMO: Os eventos climáticos extremos assumem grande relevância socioeconômica, principalmente nas regiões sensíveis climaticamente como as de clima Semiárido. A análise do regime pluviométrico de determinada região pode ser feita pelo Índice de Anomalia de Chuva (IAC), que permite classificar o regime pluviométrico quanto às anomalias anuais da precipitação, inferindo sobre os ciclos secos e úmidos. Diante disso, objetivou-se estudar a variabilidade espaço-temporal das precipitações de uma área inserida no Semiárido do Brasil. Para isso, utilizou-se o IAC para a normal climatológica 1987-2017 de 11 postos pluviométricos inseridos na mesorregião do Sertão do estado de Pernambuco. Foram analisadas as precipitações anuais e interanuais, determinadas as anomalias negativas e positivas ao longo da normal climatológica, e, por meio da interpolação espacial da precipitação pelo método interpolação de distância inversa ponderada (IDW), elaborados mapas de distribuição espacial da pluviosidade em diferentes anomalias anuais. Os resultados apontaram que em $90,9 \%$ dos postos estudados a quadra chuvosa ocorre entre os meses de janeiro a abril, e que o acumulado destes meses corresponde a mais de $50 \%$ do volume anual precipitado, demonstrando assim, a irregularidade na distribuição anual da precipitação pluvial. Verificou-se que em 55\% dos anos houve anomalias negativas na mesorregião do Sertão de Pernambuco. Outrossim, constatou-se, espacialmente, uma maior homogeneidade na distribuição espacial das anomalias negativas em relação às positivas, ou seja, os eventos de seca, quando ocorreram, tenderam a atingir toda a área estudada.

Palavras-chave: Índice de Anomalia de Chuva; Eventos Extremos; Interpolação Espacial; Pluviosidade.

ABSTRACT: Extreme weather events assume great socioeconomic relevance, especially in regions climatically sensitive such as semi-arid climate. The analysis of the rainfall regime of a given region can be made by the Rainfall Anomaly Index (RAI), which allows classifying the rainfall regime as to the annual anomalies of precipitation, inferring on the dry and humid cycles. Therefore, the objective was to study the spatial-temporal variability of precipitations of an area inserted in the Semiarid region of Brazil. For this, the RAI was used for the climatological normal 1987-2017 of 11 rainfall stations inserted in the mesoregion of the Sertão of the state of Pernambuco. The annual and interannual precipitations were analyzed, determined the negative and positive anomalies along the climatological normal, and, through the spatial interpolation of precipitation by the Method of inverse of the Square of Distance, elaborated spatial distribution maps of rainfall in different annual anomalies. The results showed that in $90.9 \%$ of the stations studied the rainy block occurs between the months of January to April, and that the accumulated of these months corresponds to more than $50 \%$ of the precipitated annual volume, thus demonstrating the irregularity in the annual distribution of rainfall. It was found that in $55 \%$ of the years there were negative anomalies in the mesoregion of the Sertão of Pernambuco. Moreover, it was spatially verified a greater homogeneity in the spatial distribution of negative

Recebido: Abril 06, 2021. Revisado: Agosto 09, 2021. Aceito: Agosto 24, 2021. 
anomalies in relation to positive anomalies, that is, drought events, when they occurred, tended to reach the entire studied area.

Keywords: Climatology; Extreme Events; Spatial Interpolation; Rainfall.

\section{INTRODUÇÃO}

O clima é o sistema da natureza que mais recebe impactos das mudanças por intervenções humanas, e um dos mais complexos e dinâmicos sistemas de se estudar, diagnosticar e interpretar suas alterações, muito em função da complexidade dos limites atmosféricos e geográficos envolvidos (Bertoni \& Tucci, 2013). Os prognósticos relacionados às mudanças climáticas indicam uma alteração do regime pluviométrico e tendência de aumento de temperatura em diversas partes do mundo (Lima et al., 2016; Silva et al., 2017a.; Chargui et al., 2018; Vinke et al., 2017) o que deverá causar alterações no ciclo hidrológico, em especial nos regimes de precipitações, de modo a aumentar a frequência de eventos extremos de chuva (Westra et al., 2014). Nesse sentido, compreender o regime de precipitação e a distribuição espaço-temporal de eventos extremos de uma região possibilita auxiliar no planejamento agropecuário, na compreensão da dinâmica das recargas de bacias hidrográficas e no planejamento contra enchentes ou secas intensas, à medida em que fornece informações importantes que podem ser usadas na elaboração de políticas públicas adequadas à realidade local.

Nesse contexto, diferentes abordagens podem ser utilizadas para caracterizar o regime de precipitação de um local em um determinado período, e uma dessas abordagens é o Índice de Anomalia de Chuva (IAC) - Rain Anomaly Index —, índice desenvolvido por Rooy \& Van (1965), que possibilita inferir sobre as anomalias negativas e positivas da precipitação durante um período. Lançando mão desse método, torna-se possível compreender as características pluviométricas de determinada região, o que, por sua vez, contribui para o entendimento do clima local e favorece a identificação de áreas suscetíveis à ocorrência de extremos climáticos (Santos et al., 2014; Nóbrega et al., 2015; Hänsel et al., 2016; Nascimento et al., 2019; Yadav et al., 2021). Por ser um método simples e com boa eficiência em comparação a índices mais complexos (Oladipo, 1985), o IAC tem sido adotado em muitos estudos desenvolvidos no sentido de verificar as anomalias de chuva em diferentes regiões (Noronha et al., 2016; Costa \& Rodrigues, 2017; Nafchi, 2018; Santos et al., 2020). Entretanto, apesar de ser muito aplicado nos estudos de eventos climáticos extremos, poucos trabalhos o relacionam à variabilidade espacial da precipitação, sobretudo, para regiões Áridas e Semiáridas.

Uma alternativa para inferir sobre a variabilidade espacial trata-se do método interpolação de distância inversa ponderada (IDW), um método simples e eficiente de interpolação espacial que têm sido amplamente aplicado em diversos estudos envolvendo fatores ambientais, como a precipitação (Yang et al., 2015; Ros \& Tosaka, 2018). Dessa forma, o IAC contextualizado com a variabilidade espacial possibilita inferir sobre a dinâmica da distribuição das chuvas de uma região. Tal investigação é extrema relevância, em especial para locais de clima semiárido, que são regiões historicamente sensíveis, do ponto de vista social, aos impactos ocasionados pela ocorrência de extremos climáticos (Marengo et al., 2016; Mattos \& May, 2020).

As regiões áridas e semiáridas correspondem a aproximadamente 55\% das terras do globo, abrangendo, por exemplo, países da América do Sul, como Argentina, Brasil e Chile (Hussain et al., 2018). No Brasil, o Semiárido possui uma área aproximada de 1,03 milhões de $\mathrm{km}^{2}$, abrangendo, em especial, a região Nordeste do país. Nesse contexto climático, está o estado de Pernambuco, que possui cerca de $87,60 \%$ da sua área inserida nesse tipo clima, e, historicamente é afetado por extremos positivos e negativos de chuva (Marengo et al., 2016), que desencadeiam problemas de ordem social, econômica e ambiental, exemplificados na ocorrência de estiagem prolongada, redução crítica no nível de reservatórios e inundações.

No Semiárido brasileiro a precipitação pluvial sofre influência de diferentes sistemas atmosféricos que estão associados a padrões de variação nas mesmas escalas de tempo nos oceanos Pacífico e Atlântico, com a variabilidade interanual associada a El Niño Oscilação Sul, ENOS, ou a Oscilação do Atlântico Norte (North Atlantic Oscillation - NAO), e a variabilidade decadal com a Oscilação Decadal do Pacífico (Pacific Decadal Oscillation - PDO), no Pacífico, e com a Oscilação Multidecadal do Atlântico (Atlantic Mutidecadal Oscillation - AMO), no Atlântico (Hastenrath, 1984). Devido a variabilidade climática que essas regiões apresentam, há uma dificuldade de proporcionar 0 avanço das atividades econômicas como agricultura e pecuária, atividades fortemente dependentes do recurso hídrico (Silva et al., 2017a). 
Diante do exposto, objetivou-se fornecer informações acerca da dinâmica espaço-temporal das anomalias de precipitação pluvial de uma região de clima semiárido, no Nordeste do Brasil, para os anos de 1987 a 2017, através do Índice de Anomalia de Chuva (IAC) e interpolação espacial da precipitação.

\section{MATERIAL E MÉTODOS}

\section{Área de Estudo}

Para esse estudo adotou-se postos pluviométricos inseridos na mesorregião do Sertão do estado de Pernambuco, Nordeste do Brasil (Figura 1). Essa mesorregião está localizada entre as coordenadas geográficas $7,35^{\circ}$ e $9,02^{\circ}$ sul; $40,795^{\circ}$ e $36,90^{\circ}$ oeste, possui uma área de $32.450 \mathrm{~km}^{2}$, abrange aproximadamente $33,0 \%$ da área do estado, subdividida em quatro microrregiões (MCR), sendo elas: Moxotó, Pajeú, Salgueiro e Araripe (Instituto Brasileiro de Geografia e Estatística, 2018).

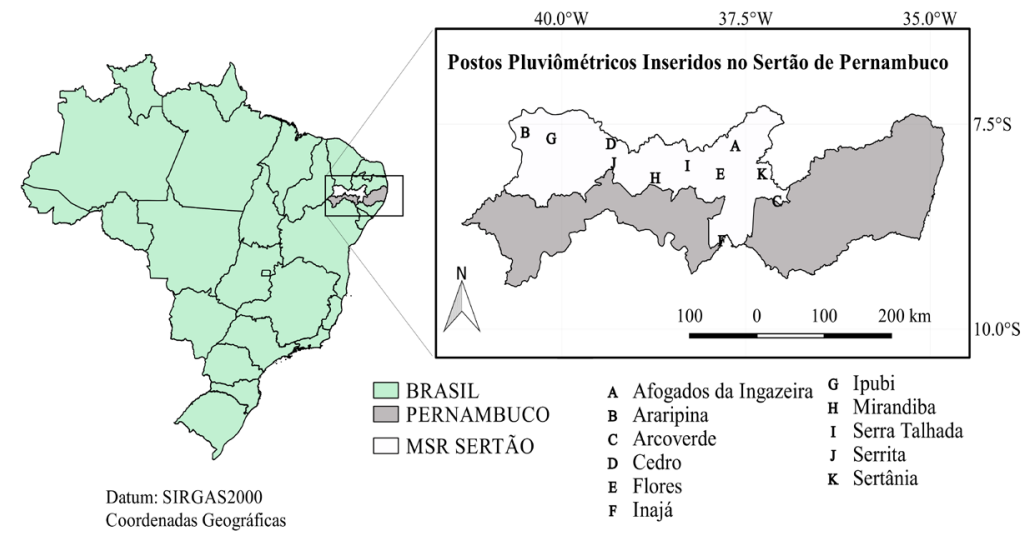

Figura 1 - Localização da Mesorregião (MSR) do Sertão de Pernambuco e a distribuição dos postos pluviométricos.

\section{Dados Pluviométricos}

Foram obtidos dados de precipitações mensais de 31 anos (1987-2017) de 11 postos pluviométricos (tabela 1) distribuídos no Sertão do estado de Pernambuco. Os dados brutos foram obtidos no Sistema de Informações Hidrológicas - HidroWEB da Agência Nacional das Águas (2019) e na plataforma digital da APAC- Agência Pernambucana de Águas e Clima (2019).

Tabela 1 - Informações dos postos pluviométricos da Agência Nacional das Águas (ANA) e da Agência Pernambucana de Águas e Climas (APAC).

\begin{tabular}{|c|c|c|c|c|c|}
\hline Agência & Estação & Código da estação & Latitude & Longitude & Falhas (\%) \\
\hline APAC & Afogados da Ingazeira & 149 & $-7,739$ & $-37,648$ & 20,7 \\
\hline APAC & Araripina & 3 & $-7,458$ & $-40,417$ & 13,4 \\
\hline APAC & Arcoverde & 82 & $-8,434$ & $-37,056$ & 10,7 \\
\hline APAC & Cedro & 6 & $-7,721$ & $-39,236$ & 21,2 \\
\hline ANA & Flores & 737027 & $-7,867$ & $-37,973$ & 12,1 \\
\hline ANA & Inajá & 837038 & $-8,921$ & $-37,831$ & 9,9 \\
\hline APAC & Ipubi & 84 & $-7,652$ & $-40,147$ & 19,8 \\
\hline APAC & Mirandiba & 54 & $-8,116$ & $-38,727$ & 13,5 \\
\hline APAC & Serra Talhada & 12 & $-7,931$ & $-38,289$ & 21,7 \\
\hline APAC & Serrita & 180 & $-7,949$ & $-39,294$ & 14,52 \\
\hline APAC & Sertânia & 253 & $-8,300$ & $-37,583$ & 24,2 \\
\hline
\end{tabular}


Cabe salientar que os dados pluviométricos obtidos são oriundos da coleta convencional de dados hidrometeorológicos, ou seja, registros diários feitos pelos observadores e técnicos das supracitadas agências.

\section{Preenchimento de Falhas e Análise de consistência dos dados}

Após a obtenção dos dados, procedeu-se com o preenchimento das falhas dos dados pluviométricos. Para isso, utilizou-se o método do Vetor Regional, desenvolvido por Hiez (1977) e aprimorado por BrunetMoret (1979). Esse método constitui uma forma de realizar preenchimentos de falhas de dados pluviométricos em níveis mensal e anual. Para isso ele se baseia em uma série cronológica de índices pluviométricos, que se originam da extração, por meio do método estatístico de máxima verossimilhança das informações dos dados dos postos estudados. A partir disso determina-se dois vetores ótimos: um vetor $\mathrm{L}$ (vetor regional coluna com " $\mathrm{x}$ " linhas) e um vetor C (vetor linha com " $y$ " colunas) cujos produtos formam uma matriz de precipitação (P) distribuída espacialmente e temporalmente (Tucci, 2009).

Nesse sentido, a estimativa da altura precipitada do mês (ou ano) i em um posto jé dada por:

$\dot{\mathrm{P}}_{\mathrm{ij}}=\mathrm{L}_{\mathrm{i}}{ }^{*} \mathrm{C}_{\mathrm{j}}$

Para o cálculo dos vetores L e C utiliza-se as seguintes equações:

$C_{j}=\frac{\sum_{i=1}^{x} L_{O} * P_{i j}}{\sum_{i=1}^{x}\left(L_{O}\right)^{2}}$, com j variando de $1 \mathrm{a} \mathrm{y}$

$L_{i}=\frac{\sum_{j=1}^{y} C_{j} * P_{i j}}{\sum_{j=1}^{y}\left(C_{j}\right)^{2}}$, com i variando de $1 \mathrm{ax}$

O desvio médio obtido pela correção de falhas utilizando o método do vetor regional pode ser calculado pela seguinte fórmula:

$\overline{\mathrm{e}}_{\mathrm{ij}}=\frac{1}{\mathrm{r}} \sum \frac{\mathrm{P}_{\mathrm{ij}}-\dot{\mathrm{P}}_{\mathrm{ij}}}{\mathrm{P}_{\mathrm{ij}}}$

Sendo Pij a precipitação real; Ṗij a precipitação estimada; ēij o desvio médio entre os valores observados e calculados; e r o número de observações no período.

Após os preenchimentos das falhas, foi verificada a consistência dos dados pelo Método da Dupla Massa. Este método foi desenvolvido pelo Geological Survey (USA), consiste em selecionar os dados dos postos que se deseja verificar a consistência, e plotar o acumulado de cada um deles em relação a um posto confiável (consistente), adotado como referência (Bertoni \& Tucci, 2013). A inconsistência pode ser identificada por mudança na declividade ou desvio brusco na reta (Oliveira et al., 2010) e, como pode ser observado na Figura 2, os dados dos postos utilizados neste estudo apresentaram-se consistentes.

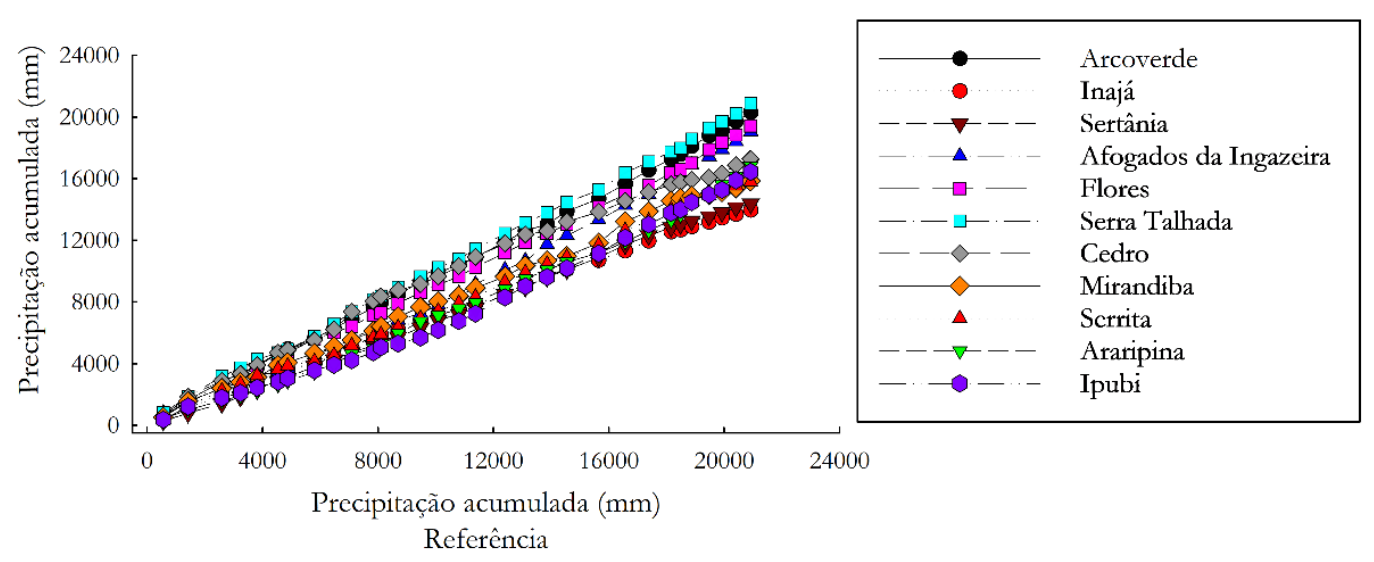

Figura 2 - Curva de dupla massa para análise de consistência dos dados pluviométricos da normal climatológica 1987-2017 de municípios inseridos no Sertão de Pernambuco. 


\section{Índice de Anomalia de Chuva (IAC) e Interpolação Espacial da Precipitação}

De posse dos dados preenchidos e analisados quanto a sua consistência, procedeu-se com o cálculo do IAC anual, conforme as Equações 1 e 2, desenvolvidas por Rooy \& Van (1965), em que:

$\mathrm{IAC}=3\left[\frac{\mathrm{n}-\mathrm{N}}{\mathrm{M}-\mathrm{N}}\right]$, para anomalias positivas

$\mathrm{IAC}=-3\left[\frac{\mathrm{n}-\mathrm{N}}{\mathrm{X}-\mathrm{N}}\right]$, para anomalias negativas

Em que:

n: precipitação (mm) observada atual (do ano que será calculado o IAC);

$\mathrm{N}$ : precipitação média da série histórica (mm);

M: média dos dez valores anuais mais altos;

$X$ : média dos dez valores anuais mais baixos.

Os IACs anuais foram ordenados em um esquema de classificação em seis categorias (Tabela 2), que varia de extremamente úmido a extremamente seco (Araújo et al., 2009). Realizou-se uma classificação da intensidade das anomalias de precipitação por posto para verificar a distribuição das anomalias de chuva durante o período estudado.

Tabela 2 - Classificação das intensidades das anomalias de precipitação anual de acordo com o Índice de Anomalia de Chuva (IAC).

\begin{tabular}{cc}
\hline IAC & CLASSIFICAÇ̃̃o \\
\hline \hline$\geq 4,00$ & Extremamente Úmido \\
\hline 2,00 a 3,99 & Muito Úmido \\
\hline 0,00 a 1,99 & Úmido \\
\hline$-2,00$ a $-0,01$ & Seco \\
\hline$-4,00$ a $-1,99$ & Muito Seco \\
\hline$\leq-4,00$ & Extremamente Seco \\
\hline
\end{tabular}

Fonte: Araújo et al. (2009)

Além disso, calculou-se o IAC médio para o Sertão de Pernambuco, envolvendo o IAC anual dos 11 postos. Após isso, e com base na classificação de intensidade das anomalias, escolheu-se para elaboração dos mapas de distribuição espacial da precipitação, seis anos de anomalias negativas e seis anos de anomalias positivas. Para as anomalias negativas escolheu-se três anos de menor valor de IAC e três anos em que enquadrasse na classificação seco para maioria dos postos. Para anomalias positivas, escolheu-se dois anos que se enquadrasse na classificação muito úmido e quatro na classificação úmida. 0 mesmo procedimento foi feito para escolha dos anos nos quais seriam gerados os mapas de anomalias positivas, ou seja, dois anos na classificação muito seco e quatro anos na classificação seco.

Nesse sentido, a interpolação espacial foi uma ferramenta para confecção de mapas de variabilidade espacial das precipitações. Para isso, a interpolação espacial das precipitações se deu pelo método de distância inversa ponderada (IDW), que consiste em um método interpolador determinístico univariado de médias ponderadas (Silva et al., 2008), em que o valor estimado num ponto interpolado é dado por:

$W i=\frac{\sum_{j=1}^{n} \frac{W_{J}}{S_{i j}{ }^{2}}}{\sum_{j=1}^{n} \frac{1}{S_{i j}{ }^{2}}}$

Em que, Wi é o valor estimado na célula interpolada; Wj é o valor do j-ésimo posto de controle; sij distância euclidiana entre o j-ésimo posto de controle e a célula interpolada.

Ao interpolar um valor para um ponto qualquer não medido, o IDW usa os valores medidos em torno do ponto a ser estimado. Desse modo, a interpolação de distância inversa ponderada (IDW) considera suposição de que os pontos próximos uns dos outros possuem maior similaridade do que 
aqueles que estão mais distantes, portanto, valores medidos mais próximos ao local de previsão têm maior influência sobre o valor previsto do que aqueles mais distantes.

Para elaboração dos mapas de distribuição espacial das precipitações em diferentes classificações, foi utilizado o software QuantumGis - QGIS - Versão 2.18 (QGIS Development Team, 2016), com o plug-in de interpolação de dados raster e, georreferenciados com o Datum SIRGAS 2000. Por seu turno, as saídas gráficas foram elaboradas no Software Sigmaplot versão 14.

\section{RESULTADOS E DISCUSSÃO}

Ao analisar a distribuição anual média da precipitação dos postos estudados (Figura 3), percebese que grande parte do volume anual precipitado se concentra entre os meses de dezembro e maio, com uma "quadra chuvosa" - quatro meses mais úmidos - que se estende, especificamente, de janeiro a abril. Entretanto, dentre os postos estudados, observou-se em um deles (Arcoverde) que o regime pluviométrico anual difere dos demais, visto que sua distribuição pluviométrica anual se apresentou menos irregular ao longo do ano, com um período chuvoso abrangendo os meses de março a julho.

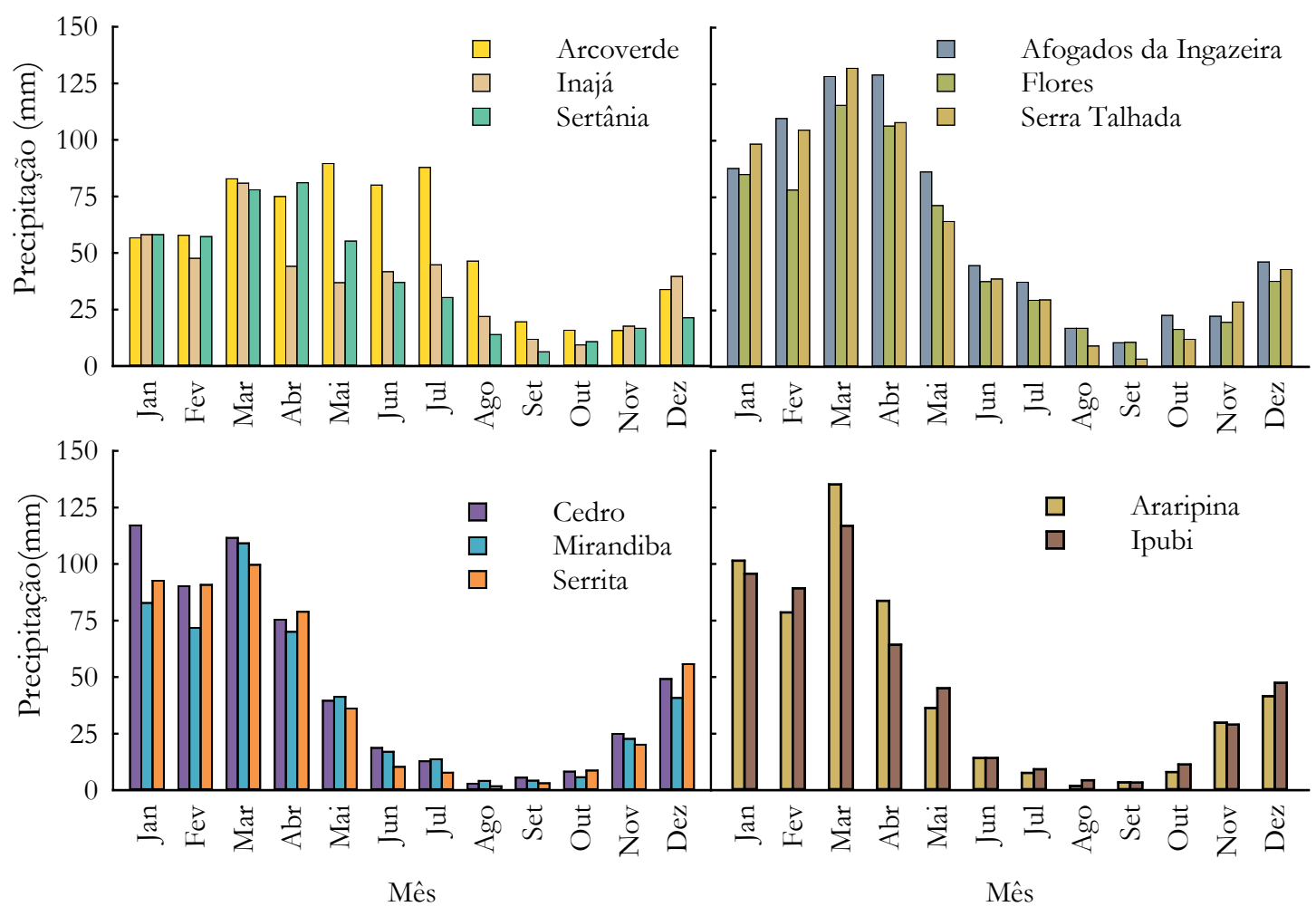

Figura 3 - Precipitação média mensal de munícipios inseridos no Sertão de Pernambuco para o período de 1987 a 2017.

Em todos os postos estudados a quadra chuvosa representa mais de $50 \%$ do regime anual. Arcoverde foi o posto que apresentou menor irregularidade na sua distribuição anual, fato evidenciado pelo acumulado dos seus cinco meses chuvosos, que representa cerca de $51 \%$ do observado durante o ano. Em contraste, alguns postos, tais como, Araripina, Cedro e Serrita, suas respectivas quadras chuvosas ultrapassaram $70 \%$ do acumulado médio anual.

Nesse aspecto da distribuição anual da precipitação no Semiárido brasileiro, Reboita et al. (2016) destacaram que a região apresenta um verão chuvoso, que compreende os meses de janeiro, fevereiro e março, com alguns valores de precipitação já em dezembro. Por sua vez, Nóbrega \& Santiago (2014) mostraram que a precipitação dessa região está ficando cada vez mais irregular, isto é, o volume anual de precipitação tem se concentrado em poucos dias do ano, em especial, na quadra chuvosa da região. Nesse sentido é importante destacar que o ciclo anual e o volume de precipitação são influenciados por diferentes sistemas meteorológicos, sendo o principal sistema a Zona de Convergência Intertropical (ZCIT), que atua em meados de janeiro a maio, ocasionando linhas de instabilidades e 
chuvas orográficas (Moura \& Vitorino, 2012) o que contribui para esse irregular distribuição temporal anual da precipitação na região.

Já na Figura 4, observa-se os valores médios de precipitação dos postos estudados. Comparando os dados de cada posto, observou-se uma proximidade entre as médias anuais de precipitação. Contudo os postos Afogados da Ingazeira, Serra Talhada, Arcoverde e Flores apresentaram médias ligeiramente superior aos demais, com precipitação média superior a $600 \mathrm{~mm}$ ano-1, enquanto Inajá, Sertânia e Mirandiba ficaram abaixo dos $500 \mathrm{~mm}$ ano-1.

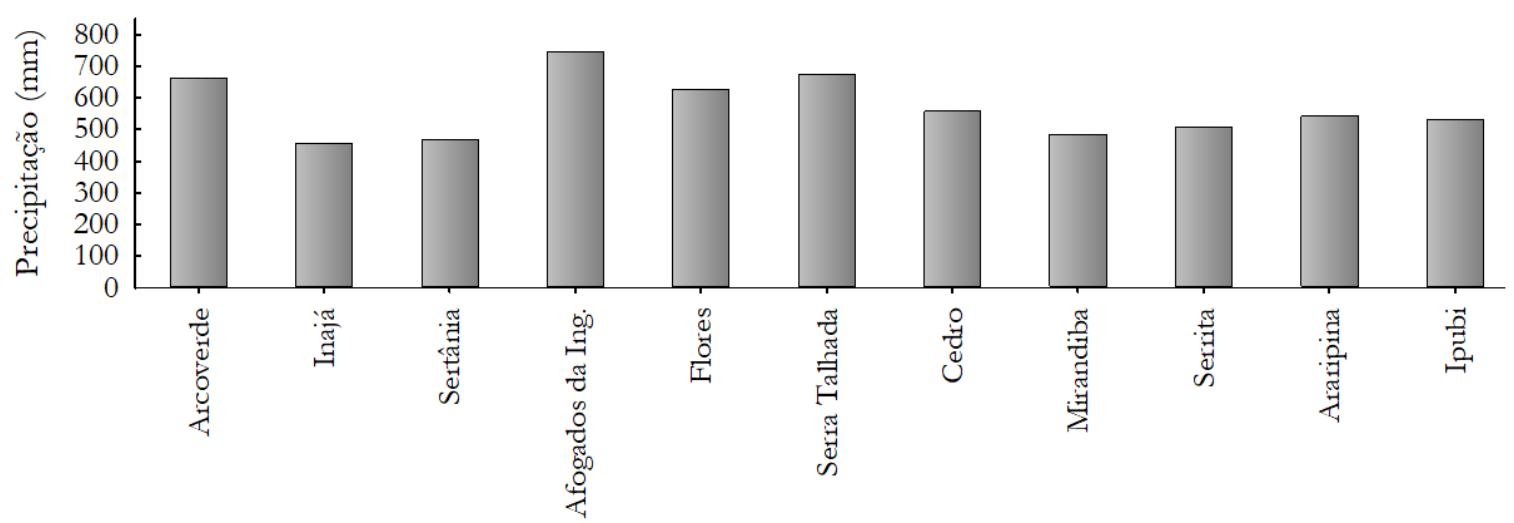

Figura 4 - Precipitação média anual de municípios do Sertão de Pernambuco, normal climatológica 19872017.

Na Figura 5 é possível conferir os totais anuais de precipitação e o IAC no decorrer do período de 1987 a 2017 nos municípios estudados. Verificou-se que durante o período estudado, nenhum dos municípios apresentaram extremos úmidos ou secos, ou seja, valores de IAC inferiores a -4 ou superiores +4 , respectivamente. Percebeu-se que os postos apresentaram uma certa semelhança entre anos chuvosos e secos. Os anos mais chuvosos compreenderam o período de 1987 a 1989, bem como, 1994 a 1998. Uma justificativa para isso pode ser o fato de que nesses anos a chuva esteve atrela a ocorrência dos fenômenos La nina (classificado como forte) ou da fase negativa do dipolo do atlântico sul, o ainda de ambos, como pode se observar nos anos de 1988 e 1989, justamente os mais chuvosos para a maioria dos postos.

Além do mais, verificou-se na maioria dos postos, ao menos 4 anos com chuva acima da média entre 2004 e 2009, intercalado por alguns anos secos como 2005 ou 2006 na maioria dos postos. Constatou-se que em anos que comprenderam os períodos de 1990 a 1993, 1998 a 2003 , e 2012 a 2017, ocorreram precipitações abaixo da média na região. A partir disso, é possível afirmar que existe uma certa semelhança na distribuição pluviométrica interanual entre os postos. Não obstante, observa-se também que a maioria dos anos de precipitação abaixo da média estiveram associados à ocorrência de El nino ou da fase positiva do dipolo do atlântico sul. No que se refere a essa diferença das médias anuais de precipitação entre os postos, pode ser justificada pela variação dinâmica atmosférica, sistemas meteorológicos e em alguns pontos, a posição geográfica que proporciona a ocorrência de fatores topoclimáticos (Thornthwaite, 1953; Marra \& Morin, 2018; Silva et al., 2018). Por seu turno, Wu et al. (2020) evidenciaram significativa relação do aumento da frequências de eventos de El Niño, no Pacífico Tropical e o período mais quente do Atlântico Norte, com a duração e intensidade dos eventos extremos de secas em todo o Nordeste do Brasil. Ademais, é importante destacar que o gradiente de precipitação do estado de Pernambuco tende a diminuir à medida em que se distância do oceano, apresentando algumas exceções, como exemplo das regiões que recebem influência da altitude (Silva et al., 2018). 

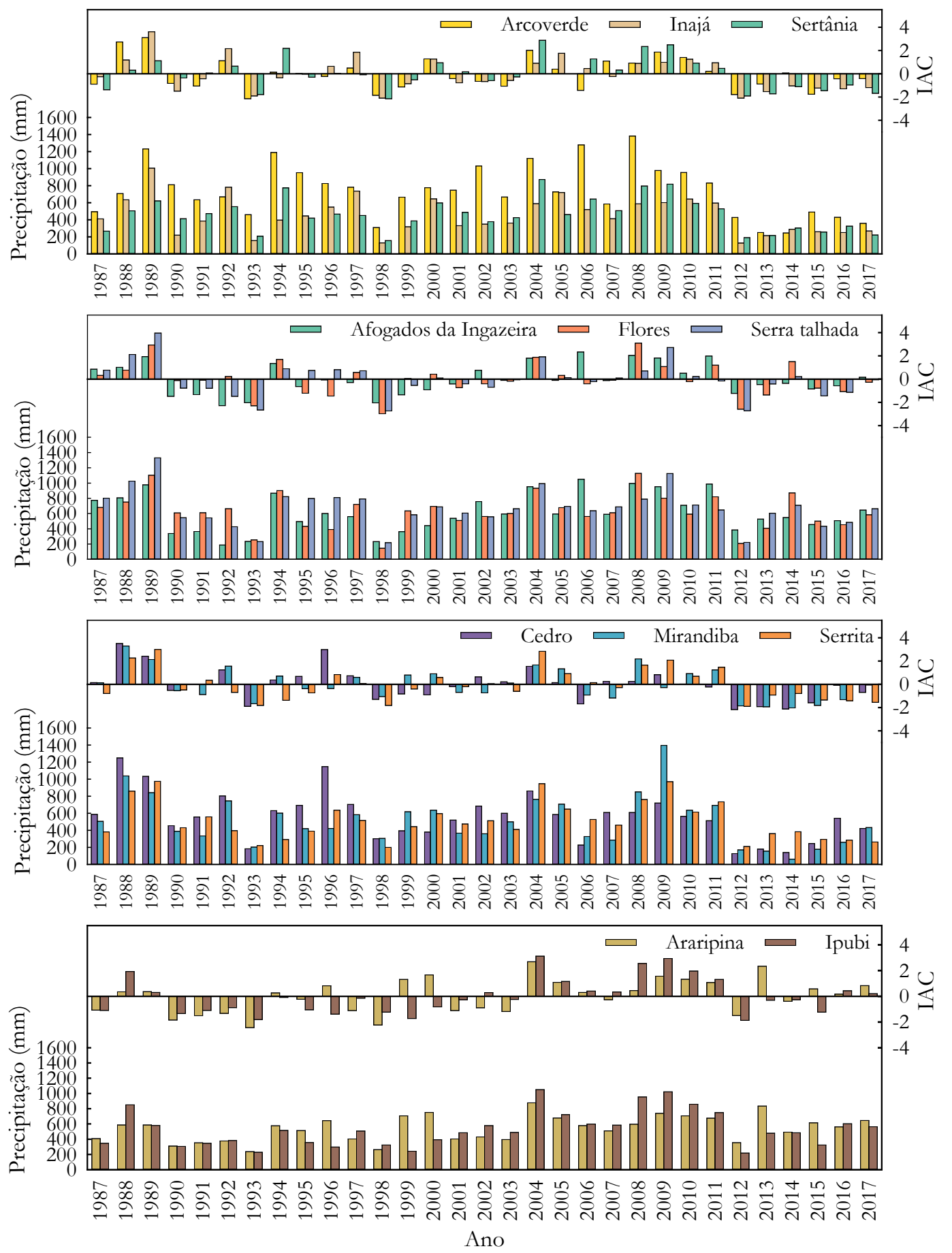

Figura 5 - Distribuição pluviométrica e Índice de Anomalia de Chuva (IAC) em postos do Sertão de Pernambuco para os anos de 1987 a 2017.

Na Figura 6, observa-se o IACs anuais para a mesorregião do Sertão de Pernambuco entre 1987 e 2017. Nota-se que os anos de maior anomalia foram 1989 e 2004, enquanto 1993, 1998 e 2012 , apresentaram baixos valores de IAC. Ademais, observou-se uma seca plurianual bem evidente, caracterizada pelas anomalias negativas, entre os anos de 2012 e 2017. 


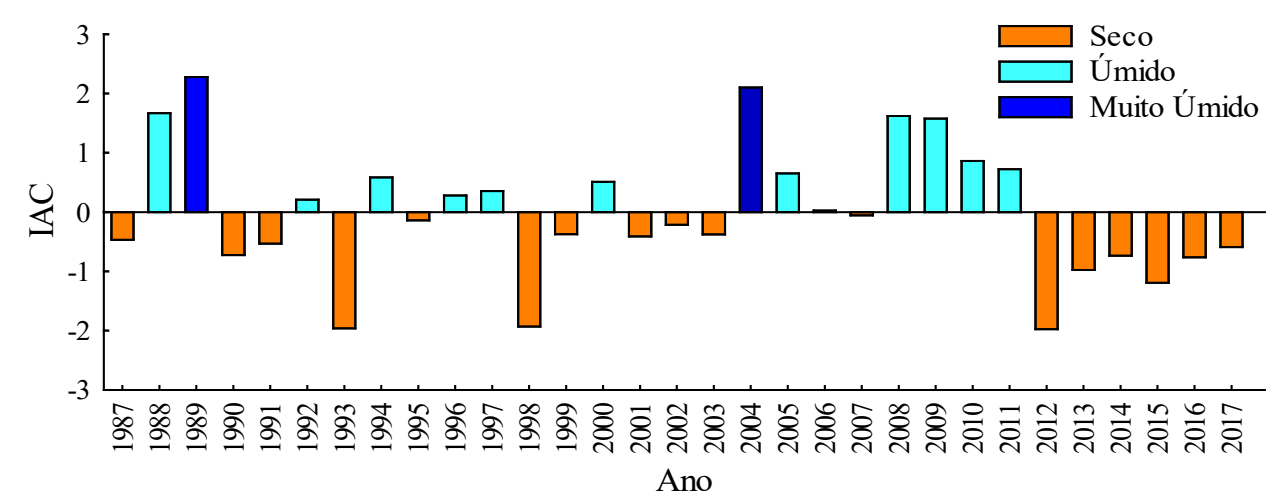

Figura 6 - Anomalias da precipitação para o Sertão de Pernambuco entre 1987 e 2017.

No que tange as anomalias anuais de chuva, Mutti et al. (2020) ao caracterizarem bacias semiáridas, também não constataram a ocorrência de anos extremamente úmidos ou extremamente secos entre 1962 e 2015 na bacia hidrográfica de Piranhas-Açu, que compreende os estados do Rio Grande do Norte e da Paraíba, Brasil. Em contraste, Santos et al. (2020) verificaram a ocorrência de eventos anuais extremos de chuva ao estudarem dados pluviométricos de 14 postos inseridos na subbacia hidrográfica do rio Piracuruca, localizada entre os estados do Ceará e do Piauí. Por sua vez, Kiros et al. (2017), em um estudo sobre os extremos de chuva em uma bacia semiárida no norte da Etiópia, obtiveram anomalias negativas em até 60,5\% dos anos (no período 1971 a 2013), para postos inseridos na bacia estudada.

Vários fatores, como a própria variabilidade espacial da precipitação, que está relacionada à fenômenos de mesoescala e fatores topoclimáticos, podem explicar a ocorrência de eventos extremos ou não em postos de pontos diferentes de uma mesma região de clima semiárido. Já em macroescala, os fatores que contribuem para o comportamento variável do volume de chuvas sobre o Semiárido do Brasil são, principalmente, os fenômenos relacionados às anomalias na temperatura da superfície oceânica do pacífico tropical e do Atlântico Sul, que afetam a posição e a intensidade dos ventos alísios e da Zona de Convergência Intertropical (ZCIT) (Hastenrath, 1984; Marengo et al., 2011; Lacerda, 2016). Nesse sentido, em anos de dipolo positivo do atlântico (i.e. anomalias positivas da temperatura da superfície do mar do Atlântico Norte) a ZCIT encontra-se mais ao norte de sua posição normal, assim, os ventos alísios de sudeste e nordeste são mais fracos, reduzindo a umidade que penetra na região Nordeste do Brasil (Nóbrega \& Santiago, 2014). Por outro lado, vários estudos indicam que há uma relação entre a ocorrência de secas no Nordeste do Brasil e o fenômeno El Niño (fases positivas do Pacífico), enquanto a La Niña está mais associada com a ocorrência de chuvas (Kane, 1989; Rodrigues et al., 2017). Um forte exemplo disso foi evidenciado no período entre 1997e 1998, em que o El Niño foi considerado o mais forte do século passado (Santos \& Mendes, 2020), resultando em anomalias extremamente negativas da precipitação em pontos do Sertão de Pernambuco (Silva et al., 2017b).

Na Tabela 3, considerando a ocorrência de ENOS e dipolo do Atlântico, observa-se que no ano de maior valor de IAC (i.e. 1989), houve o fenômeno La Niña forte associado ao dipolo negativo do atlântico, enquanto nos 3 anos mais secos (i.e. 1993,1998 e 2012) em dois deles (1998 e 2012) esteve associado à fase positiva do dipolo do atlântico. Observou-se ainda, que dos 31 anos estudados, 17 anos foram secos, e destes, nove estiveram associados à ocorrência do fenômeno El Niño, onde seis anos foram de El Niño fraco, um ano de El Niño moderado e dois anos de El Niño forte. Na Tabela 2 verifica-se ainda a ocorrência da fase positiva de dipolo do atlântico, durante seis dos sete anos de seca plurianual do período de 2012 a 2017 (Figura 6). Vale ressaltar que dentro desses seis anos, em 2015 e 2016 houve El Niño fraco e forte, respectivamente, em contraste com cinco anos de ocorrência do dipolo positivo (National Oceanic and Atmospheric Administration, 2020b), sugerindo, portanto, uma forte relação da fase positiva do dipolo do atlântico com os anos em que a região expressa anomalia negativa na precipitação. Quando se verifica as anomalias positivas (IAC >0,0), é possível verificar que do período analisado, ocorreram concomitantemente La Niña e fase negativa do dipolo em oito anos, e destes, em sete a região do Sertão apresentou anomalia positiva da precipitação. Dos 31 anos, em 17 houve IAC negativo da precipitação, sendo oito anos associados ao dipolo positivo do atlântico.

Tabela 3 - Ocorrência e intensidade de ENOS e Dipolo do atlântico no período 1987 a 2017. 


\begin{tabular}{|c|c|c|c|c|c|c|}
\hline \multicolumn{4}{|c|}{ Ocorrências de El Niño } & \multicolumn{3}{|c|}{ Ocorrências de La Niña } \\
\hline Fraco & Moderado & Forte & Muito forte & Fraco & Moderado & Forte \\
\hline 2004-05 & $1986-87$ & $1987-88$ & $1997-98$ & $2005-06$ & $1995-96$ & $1988-89$ \\
\hline 2006-07 & 1994-95 & 1991-92 & $2015-16$ & 2009-09 & 2011-12 & 1998-99 \\
\hline \multirow[t]{3}{*}{ 2014-15 } & 2002-03 & & & $2016-17$ & & 1999-00 \\
\hline & $2009-10$ & & & & & 2007-08 \\
\hline & & & & & & $2010-11$ \\
\hline \multicolumn{7}{|c|}{ Dipolo do Atlântico } \\
\hline \multicolumn{4}{|c|}{ Fase positiva } & \multicolumn{3}{|c|}{ Fase negativa } \\
\hline \multicolumn{4}{|c|}{$\begin{array}{c}\text { 1990; 1992; } 1997 ; 1998 ; 2005 ; 2007 ; 2012 ; \\
2013 ; 2015 ; 2016 ; 2017\end{array}$} & \multicolumn{3}{|c|}{$\begin{array}{c}1988 ; 1989 ; 1991 ; 1994 ; 1995 ; 1996 ; 1999 ; 2000 ; 2002 ; \\
2003 ; 2008 ; 2009 ; 2011 ; 2014\end{array}$} \\
\hline
\end{tabular}

A Figura 7 mostra a variabilidade espacial e temporal da precipitação em anos secos, (1987, 2003 e 2014) muito secos (1993, 1998 e 2012) na região estudada. Referente aos anos muito secos, cujas médias anuais de precipitação foram 250,8 mm; 245,3 mm e 248,1 mm, respectivamente, observouse uma homogeneidade na distribuição espacial da precipitação em quase toda a região. Entretanto, verificou-se na parte a nordeste da mesorregião, que nos três anos classificados como muito secos, houve valores de precipitação próximo a faixa de $530 \mathrm{~mm}$, enquanto a parte central e oeste, os valores observados foram entre 200 e $310 \mathrm{~mm}$, nessa ordem.
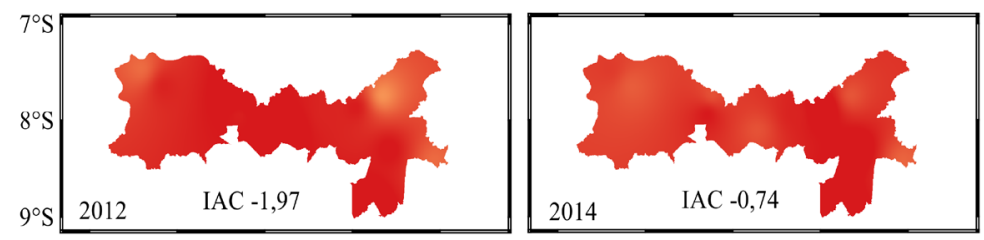

Preciptação (mm)
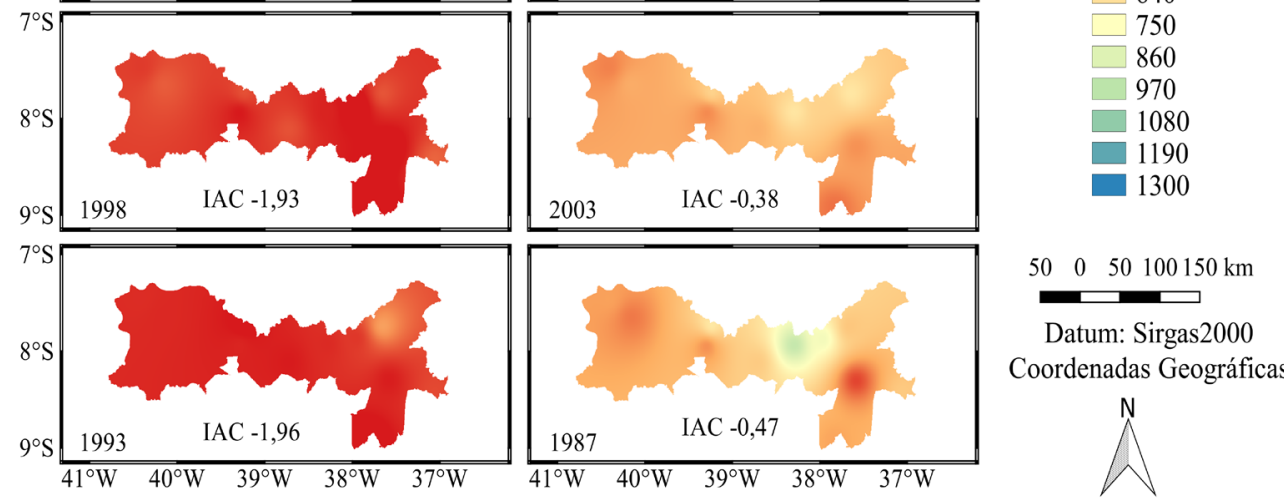

Figura 7 - Anos com Índice de Anomalia de Chuva (IAC) negativo para o Sertão de Pernambuco.

Nos anos 1987, 2003 e 2014, observou-se uma diferença na distribuição espacial da precipitação. No ano de 1987, em que o IAC foi de -0,41 e média de precipitação 527,9 mm, percebeu-se uma zona úmida no centro-norte do Sertão, onde as precipitações foram superiores ao restante da mesorregião. Essa zona de maior precipitação, refere-se aos municípios da microrregião do Pajeú, que apesar do Sertão ter predominado a classificação seca, postos como Serra Talhada e Flores, no ano 1987, observou-se chuvas acumuladas de $801,6 \mathrm{~mm}$ e $680,2 \mathrm{~mm}$, e IAC de 0,77 e 0,32, respectivamente.

Ainda na Figura 7, observou-se para os anos de 2003 e 2014 uma distribuição mais homogênea. Porém, apesar de ambos os anos terem se enquadrado na classificação seco, verificou-se no ano 2014 um grau de severidade de seca maior que em 2003, visto que o primeiro apresentou IAC igual a -1,1, e o segundo igual a -0,46. Ressalta-se que nesses dois anos houve influência de El Niño, sendo caracterizado como moderado em 2003 e fraco em 2014 (National Oceanic and Atmospheric Administration, 2020a).

A Figura 8, mostra a distribuição da pluviosidade em anos cujas anomalias positivas foram as mais altas no período estudado. No ano de 1988, o IAC médio para a mesorregião foi de 1,98, enquanto 
2005 foi 0,51 e em 2009 foi de 0,68. Os valores de IAC para os anos de 2004, 2009 e 1989, foram iguais a $2,29,2,51$ e 2,27 , respectivamente.
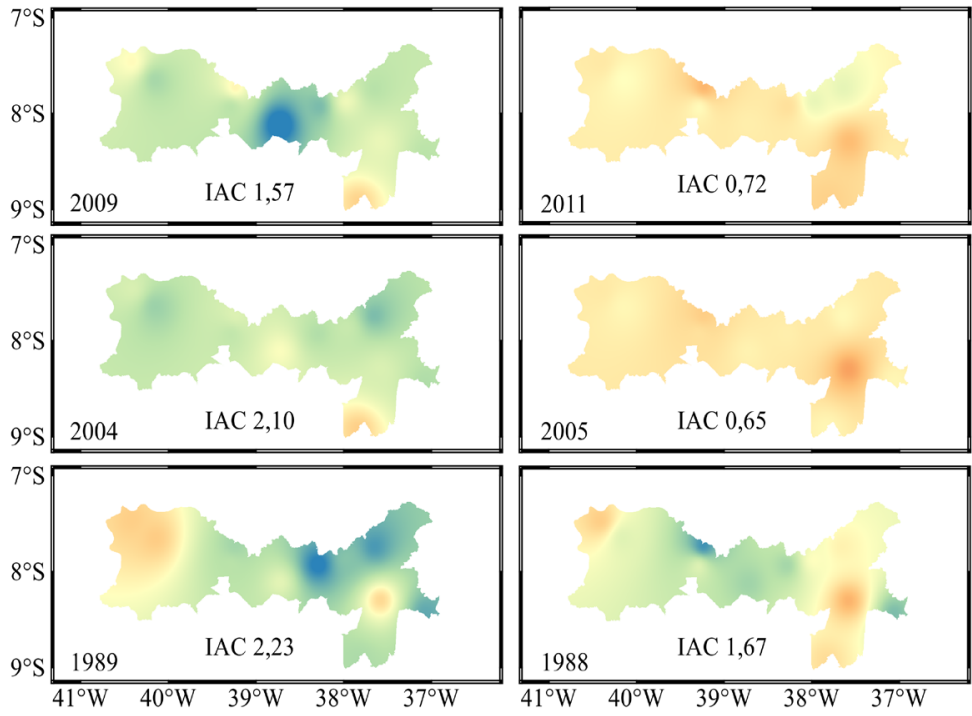

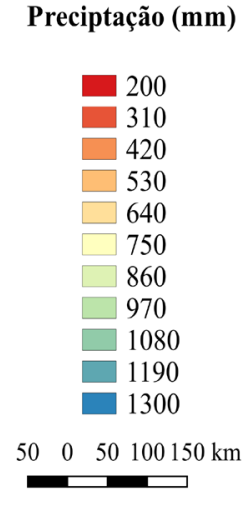

Datum: Sirgas 2000 Coordenadas Geográficas

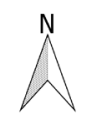

Figura 8 - Anos classificados como muito úmidos ou úmidos pelo Índice de Anomalia de Chuva (IAC) para o Sertão de Pernambuco.

Percebe-se nas imagens que a precipitação é mais irregular em anos com anomalias positivas, mas com uma tendência de quantidades maiores de chuva na região central até nordeste da mesorregião, que coincide com a microrregião do Pajeú. Vale ressaltar que no ano 1988 houve a influência do fenômeno La Niña com intensidade forte, enquanto em 2005, houve influência de La Niña fraco, e em 2011 La Niña Moderado (National Oceanic and Atmospheric Administration, 2020a). Por sua vez, 1989 esteve sob influência do fenômeno La Niña, cuja intensidade foi considerada forte, ao passo que o ano de 2004, observou-se influência de El Niño fraco, e em 2009, La Niña fraco.

Contudo, os fenômenos El Niño e La Niña e o dipolo do Atlântico não são capazes, isoladamente, de explicar as anomalias positivas e negativas de precipitação no Semiárido, ou seja, a interação desses fenômenos com os sistemas meteorológicos como a ZCIT, os Vórtice Ciclônico de Altos Níveis (VCAN), brisas marinhas, variação da pressão atmosférica e altitude, e relevo é que são determinantes no regime e variabilidade de chuvas do Nordeste do Brasil, além da atuação de sistemas convectivos e massas de ar predominantes na região (Andreoli \& Kayano, 2007; Marengo et al., 2011; Carvalho \& Oyama, 2013; Reboita et al., 2016; Silva et al., 2018). Nesse sentido, no que diz respeito aos VCANs, apesar de poucos trabalhos relatarem sua influência, por exemplo, na variação espaço-temporal da precipitação, Diniz \& Pereira (2015) relataram que este contribui para a variação espacial da precipitação no Nordeste do Brasil e, por conseguinte, no Sertão de Pernambuco. Para esses autores, na medida em que os VCANs se formam no Atlântico e avançam por todo interior do Nordeste, estes produzem nuvens de chuva em sua periferia e ar seco em seu centro, formando localmente e, de forma temporária, áreas de altas pressões, que podem contribuir para ocorrência de chuvas em qualquer parte do estado, conforme sua posição, que é variável.

Portanto, os resultados aqui constatados podem contribuir quando da elaboração de projetos para setores como agricultura, pecuária, abastecimento e indústria, setores diretamente relacionados ao regime pluviométrico e que diretamente influenciam a economia e as questões sociais de um local.

\section{CONCLUSÕES}

Os quatro meses mais chuvoso do Sertão de Pernambuco, Nordeste do Brasil, contribuem com mais de $50 \%$ do volume anual de chuvas. Em escala interanual, a distribuição espacial das anomalias na região é bastante homogênea nos anos muito secos e heterogênea em anos muito chuvosos, resultando em classificações anuais de anomalias de precipitação diferentes entre os postos, num mesmo ano. A região apresenta uma alta variabilidade pluviométrica temporal, com uma alternância de períodos mais chuvosos e secos, porém com a predominância de anomalias negativas. Apesar disso, 
não se constatou para o período estudado a ocorrência de anomalias extremas de precipitação, ou seja, anos extremamente secos ou extremamente úmidos. Apesar de a não constatação de eventos extremos, ressalta-se a ocorrência de anos consecutivos de anomalias negativas, evidenciando a ocorrência de secas plurianuais, ainda que não caracterizadas como extremas de acordo com o IAC. Portanto, acredita-se que tais peculiaridades evidenciadas nesse estudo, como a distribuição irregular da chuva ao longo do ano e a ocorrência de anomalias negativas consecutivas anuais de chuvas, devem ser levadas em consideração por gestores e técnicos que visem a elaboração de projetos ambientais ou agropecuários para a região.

\section{REFERÊNCIAS}

Agência Nacional das Águas - ANA. (2019). Séries Históricas. Sistema de informações hidrológicas. Recuperado em 19 de setembro, de http://www.snirh.gov.br/hidroweb/serieshistoricas

Agência Pernambucana de Águas e Clima - APAC. (2019). Monitoramento Pluviométrico. Recuperado em 19 de setembro, de http://old.apac.pe.gov.br/meteorologia/monitoramento-pluvio.php

Andreoli, R.V., \& Kayano, M.T.A. (2007). Importância relativa do Atlântico Tropical Sul e Pacífico leste na variabilidade de precipitação do Nordeste do Brasil. Revista Brasileira de Meteorologia, 22 (1), 6374.

Araújo, L. E., Moraes Neto, J. M., \& Souza, F. A. S. (2009). Análise climática da bacia do rio Paraíba Índice de Anomalia de Chuva (IAC). Revista de Engenharia Ambiental, 6(3), 508-523.

Bertoni, J. C., \& Tucci, C. E. M. (2013). Precipitação. In C. E. M. Tucci (Org.), Hidrologia: ciência e aplicação. (pp. 176). Porto Alegre: Editora da Universidade Federal do Rio Grande do Sul.

Brunet-Moret, Y. (1979). Homogéneisation des précipitacions. Cahiers ORSTOM, Série Hydrologie, 3(4), 147-170.

Carvalho, M.A.V., \& Oyama, M.D. (2013). Variabilidade da largura e intensidade da Zona de Convergência Intertropical atlântica: aspectos observacionais. Revista Brasileira de Meteorologia, 28(3), 305-331.

Chargui, S., Jaberi, A., Cudennec, C., Lachaal, F., Calvez, R., \& Slimani, M. (2018). Statistical detection and no-detection of rainfall change trends and breaks in semiarid Tunisia: 50 years over the Merguellil agro-hydro-climatic reference basin. Arabian Journal of Geosciences, 134, 675-689. http://dx.doi.org/10.1007/s12517-018-4001-9

Costa, J. A., \& Rodrigues, G. P. (2017). Space-time distribution of rainfall anomaly index (RAI) for the Salgado Basin, Ceará State - Brazil. Ciência e Natura, 39(3), 627-634.

Diniz, M. T. M., \& Pereira, V. H. C. (2015). Climatologia do estado do Rio Grande do Norte, Brasil: sistemas atmosféricos atuantes e mapeamento de tipos de clima. Boletim Goiano de Geografia, 35(3), 488-506. http://dx.doi.org/10.5216/bgg.v35i3.38839

Hänsel, S., Schucknecht, A., \& Matschullat, J. (2016). The Modified Rainfall Anomaly Index (mRAI): is this an alternative to the Standardised Precipitation Index (SPI) in evaluating future extreme precipitation characteristics? Theoretical and Applied Climatology, 123, 827-844. http://dx.doi.org/10.1007/s00704-015-1389-y

Hastenrath, S. (1984). Interannual variability and annual cycle: mechanisms of circulation and climate in the tropical Atlantic. Monthly Weather Review, 112, 1097-1107.

Hiez, G. L. (1977). Homogenéité des données pluviométriques. Cahiers ORSTOM. Série Hydologie, 14(2), 129-172.

Hussain, J., Khaliq, T., Ahmad, A., Akhter, J., \& Asseng, S. (2018). Wheat responses to climate change and its adaptations: a focus on arid and semi-arid environment. International Journal of Environmental of Research, 12(1), 117-126.

Instituto Brasileiro de Geografia e Estatística - IBGE. (2018). Censo demográfico. Recuperado em 29 de agosto, de https://www.ibge.gov.br/cidades-e-estados/pe/.html?

Kane, R. P. (1989). Relationship between the southern oscillation/El Niño and rainfall in some tropical and midlatitude regions. Proceedings of the Indiana Academy of Sciences, 98(3), 223-235. http://dx.doi.org/10.1007/BF02881825 
Kiros, G., Shetty, A., \& Nandagiri, L. (2017). Extreme rainfall signatures under changing climate in semiarid northern highlands of Ethiopia. Cogent Geoscience, 3(1), 1353719. http://dx.doi.org/10.1080/23312041.2017.1353719

Lacerda, F. F. (2016). Tendências de temperatura e precipitação e cenários de mudanças climáticas de longo prazo no Nordeste do Brasil e em ilhas oceânicas (Tese de doutorado). Programa de Pósgraduação em Engenharia Civil, Centro de Tecnologia e Geociências, Universidade Federal de Pernambuco, Recife.

Lima, J. S. S., Silva, S. A., Bernardes, P. M., Fonseca, A. S., \& Pereira, J. M. S. (2016). Variabilidade espacial dos percentis 75 da precipitação pluvial mensal no estado do espírito santo. Engenharia na Agricultura, 24, 393-405. http://dx.doi.org/10.13083/ reveng.v24i5.700

Marengo, J. A., Alves, L. M., Beserra, E. A., \& Lacerda, F. F. (2011). Variabilidade e mudanças climáticas no semiárido brasileiro (pp. 410-449). Campina Grande: Instituto Nacional do Semiárido.

Marengo, J. A., Cunha, A. P., \& Alves, L. M. (2016). A seca de 2012-15 no semiárido do Nordeste do Brasil no contexto histórico. Climanálise, 3(1), 1-6.

Marra, F., \& Morin, E. (2018). Autocorrelation structure of convective rainfall in semiarid-arid climate derived from high-resolution X-Band radar estimates. Atmospheric Research, 200, 126-138. http://dx.doi.org/10.1016/ j.atmosres.2017.09.020

Mattos, L. C., \& May, P. (2020). Duas secas climaticamente análogas no semiárido nordestino com impactos sociais distintos. Desenvolvimento e Meio Ambiente, 55, 28-23. http://dx.doi.org/10.5380/dma.v55i0.73796

Moura, M. N., \& Vitorino, M. I. (2012). Variabilidade da precipitação em tempo e espaço associada à Zona de Convergência Intertropical. Revista Brasileira de Meteorologia, 27(4), 475-483. http://dx.doi.org/10.1590/S0102-77862012000400010

Mutti, P. R., Abreu, L. P., Andrade, L. M. B., Spyrides, M. H. C., Lima, C. K., Oliveira, C. P., Dubreuil, V., \& Bezerra, B. G. (2020). Uma estrutura detalhada para a caracterização da climatologia das chuvas em bacias semiáridas. Theoretical and Applied Climatology, 137(1), 109-125.

Nafchi, R. A. (2018). Analysis of annual precipitation and water table changes in shahrekord aquifer. International Journal of Current Microbiology and Applied Sciences, 7(5), 560-568. http://dx.doi.org/10.20546/ ijcmas.2018.705.070

Nascimento, M.B., Silva, J.M., Souza Clemente, T., \& Araújo, L. E. (2019). Análise espaço-temporal da precipitação no município de Cabaceiras no Estado da Paraíba. Revista Eletrônica de Gestão e Tecnologias Ambientais, 7(2), 176-187. http://dx.doi.org/10.9771/ gesta.v7i2.30788

National Oceanic and Atmospheric Administration - NOAA. (2020a). Climate Prediction Center. Historical El Nino / La Nina episodes (1950-2020). Recuperado em 17 de agosto de 2020, de https://origin.cpc.ncep.noaa.gov/products/analysis_monitoring/ensostuff/ONI_v5.php

National Oceanic and Atmospheric Administration - NOAA. (2020b). Climate Prediction Center. North Atlantic Oscilation (NAO) (1950-2020). Recuperado em 17 de agosto de 2020, de https://www.cpc.ncep.noaa.gov/products/precip/CWlink/pna/norm.nao.monthly.b5001.current.as cii

Nóbrega, R. S., \& Santiago, G. A. C. F. (2014). Tendência de temperatura na superfície do mar nos oceanos Atlântico e Pacífico e variabilidade de precipitação em Pernambuco. Mercator (Fortaleza), 13(1), 107-118.

Nóbrega, R. S., Farias, R. F. L., \& Santos, C. A. C. (2015). Variabilidade temporal e espacial da precipitação pluviométrica em Pernambuco através de índices de extremos climáticos. Revista Brasileira de Meteorologia, 30(2), 171-180. http://dx.doi.org/10.1590/0102-778620130624

Noronha, G. C., Hora, M. A. G. M., \& Silva, L. P. (2016). Análise do Índice de Anomalia de Chuva para a Microbacia de Santa Maria/Cambiocó, RJ. Revista Brasileira de Meteorologia, 31(1), 74-81. http://dx.doi.org/10.1590/0102-778620140160

Oladipo, E. O. (1985). A comparative performance analysis of three meteorological drought indices. International Journal of Climatology, Chichester, 5(6), 655-664. http://dx.doi.org/10.1002/joc.3370050607

Oliveira, L. F. C., Fioreze, A. P., Medeiros, A. M. M., \& Silva, M. A. S. (2010). Comparação de metodologias de preenchimento de falhas de séries históricas de precipitação pluvial anual. Revista Brasileira de Engenharia Agrícola e Ambiental, 14(11), 1186-1192. 
QGIS Geographic Information System. (2019). Information System. Open Source Geospatial Foundation Project. Recuperado em 17 de agosto de 2019, de http://www.qgis.org/

Reboita, M. S., Rodrigues, M., Armando, R. P., Freitas, C., Martins, D., \& Miller, G. (2016). Causas da semiaridez do Sertão nordestino. Revista Brasileira de Climatologia, 19(2), 254-277.

Rodrigues, L. O., Souza, W. M., Oliveira, C. V. S., \& Pereira, M. L. T. (2017). Influência dos eventos de El Niño e La Niña no regime de precipitação do Agreste de Pernambuco. Revista Brasileira de Geografia Física, 10(6), 1995-2009.

Rooy, M. P., \& Van, A. (1965). Rainfall Anomaly Index Independent of Time and Space. Notes, 14, 1-43.

Ros, F. C., \& Tosaka, H. (2018). Analysis of rainfall distribution in Kelantan River basin, Malaysia. In International Conference on Civil \& Environmental Engineering (Vol. 34, pp. 02020). https://doi.org/10.1051/e3sconf/20183402020.

Santos, F. A., Mendes, L. M. S., \& Brito da Cruz, M. L. (2020). Avaliação de ocorrências de eventos climáticos extremos na sub-bacia hidrográfica do rio Piracuruca. GEOgrafias, 28(1), 43-61.

Santos, L. S., \& Mendes, L. A. (2020). Influência da oscilação da temperatura do oceano pacífico equatorial no regime de precipitação da região hidrográfica do Atlântico Leste. Revista Brasileira de Geografia Física, 13(4), 1502-1518.

Santos, W. O., Silva, K. B., Coelho, D. C. L., Silva, K. M. P., Sobrinho, J. E., Silva, P. C. M., \& Batista, R. O. (2014). Variabilidade espacial e temporal das precipitações para a Microrregião de Pau dos Ferros-RN. Revista Brasileira de Geografia Física, 7(3), 434-441.

Silva, R. O. B., Montenegro, S. M. G. L., \& Souza, W. M. (2017a). Tendências de mudanças climáticas na precipitação pluviométrica nas bacias hidrográficas do estado de Pernambuco. Engenharia Sanitaria e Ambiental, 22(3), 579-589.

Silva, A. R., Santos, T. S., Queiroz, D. E., Gusmão, M. O., \& Silva, T. G. F. (2017b). Variações no índice de anomalia de chuva no semiárido. Journal of Environmental Analysis and Progress, 2(4), 377-384.

Silva, M. J., Queiroz, M. G., Ferraz Jardim, A. M. R., Araújo Júnior, G. N., \& Silva, T. G. F. (2018). Gradientes pluviométricos do estado de Pernambuco: uma análise do litoral ao Semiárido. Revista Engenharia na Agricultura, 26(3), 240-249. http://dx.doi.org/10.13083/ reveng.v26i3.889

Silva, S. A., Lima, J. S. S., Souza, G. S., \& Oliveira, R. B. (2008). Avaliação de interpoladores estatísticos e determinísticos na estimativa de atributos do Solo em agricultura de precisão. Idesia, 26(2), 75-81.

Thornthwaite, C. W. (1953). Topoclimatology. In Proceedings of the Toronto Meteorological Conference (227232). Royal Meteorological Society.

Tucci, C. E. M. (2009). Hidrologia: ciência e aplicação (4. ed., 943 p.). Porto Alegre: Editora da UFRGS/ABRH.

Vinke, K., Martín, M., Adams, S., Baarsch, F., \& Bondeau, A., Coumou, D., Donner, R. V., Menon, A., Perrette, M., Rehfeld, K., Robinson, A., Rocha, M., Schaeffer, M., Schwan, S., Serdeczny, O., \& Svirejeva-Hopkins, A. (2017). Climatic risks and impacts in South Asia: extremes of water scarcity and excess. Regional Environmental Change, 17(6), 1569-1583. http://dx.doi.org/10.1007/s10113-015-0924-9

Westra, S., Fowler, H., Evans, J., Alexander, L., Berg, P., Johnson, F., Kendon, E., Lenderink, G., \& Roberts, N. (2014). Future changes to the intensity and frequency of short-duration extreme rainfall. Geophys, 52, 522555. http://dx.doi.org/10.1002/2014RG000464

Wu, H., Zou, Y., Alves, L. M., Macau, E. E. N., Sampaio, G., \& Marengo, J. A. (2020). Uncovering episodic influence of oceans on extreme drought events in Northeast Brazil by ordinal partition network approaches. Chaos: An Interdisciplinary Journal of Nonlinear Science, 30(5), 053104.

Yadav, S. M., Bhagat, S. R., \& Yadav, V. G. (2021). Análise temporal da precipitação em Saurashtra, Kutch, e Diu sub-divisão da região da Índia Ocidental. Theoretical and Applied Climatology, 144, 521-533. http://dx.doi.org/10.1007/s00704-021-03564-6

Yang, X., Xie, X., Liu, L., Ji, F., \& Wang, L. (2015). Spatial interpolation of daily rainfall data for local climate impact assessment over greater sydney region. Advances in Meteorology, 563629, http://dx.doi.org/10.1155/2015/563629 


\section{Contribuições dos autores:}

Sidney Anderson Teixeira da Costa: redação, processamento dos dados, elaboração das figuras e discussão dos resultados.

Alan Cezar Bezerra: idealização e orientação, processamento dos dados.

Athos Murilo Queiroz de Araújo: obtenção dos dados, preenchimento de falhas, análise de consistência e melhoria na redação.

Michelle Ferreira da Silva: obtenção dos dados, demonstração e interpretação dos resultados e melhoria na redação;

José Francisco da Cruz: processamento dos dados e interpolação espacial;

Rafael Mateus Alves: obtenção e análise dos dados, elaboração de figuras e tabelas, melhoria na redação final;

Luciana Sandra Bastos de Souza: orientação, análise de consistência e melhoria na redação final. 